Research Paper

\title{
The effect of co-transplantation of nerve fibroblasts and Schwann cells on peripheral nerve repair
}

\author{
Yang Wang ${ }^{1 *}$, Dong Li ${ }^{2 *}$, Gangyang Wang ${ }^{1}$, Lulu Chen ${ }^{3}$, Jun Chen ${ }^{1}$, Zhangyin Liu ${ }^{4}$, Zhaofeng Zhang ${ }^{1}$, Hua \\ Shen $^{1 凶}$, Yuqing Jin ${ }^{1 凶}$, Zunli Shen ${ }^{1 \bowtie}$ \\ 1. Department of Plastic and Reconstructive Surgery, Shanghai General Hospital, Shanghai Jiao Tong University School of Medicine, Shanghai, People's \\ Republic of China; \\ 2. Department of Plastic and Reconstructive Surgery, Shanghai 9th People's Hospital, Shanghai Jiao Tong University School of Medicine, Shanghai, People's \\ Republic of China; \\ 3. Plastic Surgery Hospital, Chinese Academy of Medical Sciences \& Peking Union Medical College, Beijing, People's Republic of China; \\ 4. Jiangpu Primary Health Service Center, Shanghai, People's Republic of China. \\ * Yang Wang and Dong Li contributed equally to this work. \\ $\triangle$ Corresponding author:
}

(c) Ivyspring International Publisher. This is an open access article distributed under the terms of the Creative Commons Attribution (CC BY-NC) license (https://creativecommons.org/licenses/by-nc/4.0/). See http://ivyspring.com/terms for full terms and conditions.

Received: 2017.07.18; Accepted: 2017.09.10; Published: 2017.11.02

\begin{abstract}
Combinations of fibroblasts (Fbs) and corresponding epithelial cells have been widely used in many tissues, such as the skin and breast tissues, to augment tissue repair and remodeling. Recently, a large amount of new data has indicated that nerve Fbs play critical roles in Schwann cells (SCs) and axons in vitro. However, little is known regarding the effects of co-transplanting nerve Fbs and SCs on peripheral nerve repair in vivo. The aim of this study was to investigate the effect of co-transplanting sciatic nerve Fbs (SN-Fbs) and sciatic nerve SCs (SN-SCs) on nerve regeneration. We developed a $5 \mathrm{~mm}$ nerve-defect model in mice using a polyurethane (PUR) catheter and then injected one of four different mixtures of cells into the catheters to form the following four groups: pure Matrigel (Control group), SN-Fbs alone (SN-Fb group), SN-Fbs combined with SN-SCs at a ratio of 1:2 (Fb\&SC group) and SN-SCs alone (SN-SC group). Histological and functional analyses were performed 3 months later. The results indicated that in vitro, the expression levels of NGF, BDNF and GDNF were significantly higher, and in vivo, a more moderate amount of extracellular matrix was produced in the Fb\&SC group than in the SN-SC group. Compared to the other groups, co-transplanting SN-Fbs with SCs at a 1:2 ratio had significantly positive effects on nerve regeneration and functional recovery.
\end{abstract}

Key words: fibroblasts, Schwann cells

\section{Introduction}

Peripheral nerve defects remain an intractable challenge for researchers and always result in functional loss [1-3]. Peripheral nerve tissue engineering, in which nerve conduits and seeding cells such as Schwann cells (SCs), Schwann-like cells and stem cells are used, is currently considered a promising method [4-7]. Therefore, applications aimed at developing ideal seeding cells are a high priority in peripheral nerve regeneration research.

Peripheral nerves are composed mainly of fibroblasts (Fbs) and SCs. Recently, studies have shown that a subset of nerve Fbs originate from neural crest, similar to SCs [8]. A great deal of new data has shown that nerve Fbs increase SC migration [9-12] in vitro. When nerve Fbs are co-cultured with SCs and neurons, collagen fibril and basal lamina deposition and the extent of neurite ensheathment are clearly increased [13]. Subsequent studies revealed that Fbs stimulate basal laminin deposition and elongate SCs even in the absence of neurons [14], which resemble those observed in the initial regenerating peripheral nerves. 
Indeed, previous studies [15-17] have demonstrated that reciprocal interactions between $\mathrm{Fbs}$ and corresponding epithelial cells play critical roles in the augmentation of tissue repair and remodeling. Compared to keratinocytes alone, co-cultures of keratinocytes and dermal fibroblasts more optimally differentiated and more closely approached the characteristics of skin $[18,19]$. The notion that Fbs have been incorporated into tissue-engineered skin substitutes suggests a variety of clinical applications that could be used to promote wound healing and enhance re-epithelialization [16, 20]. Moreover, co-transplanting mammary stromal fibroblasts with mammary epithelial cells resulted in more normal epithelial structures and less neoplasia than was observed when mammary epithelial cells alone were applied [15].

In nerves, SCs share many similarities with epithelial cells, including the possession of a basement membrane. However, few previous studies have focused on the interactions between and the effects of combinations of Fbs and SCs in nerve regeneration.

In normal peripheral nerves, the ratio of $\mathrm{Fbs}$ to SCs is 1:2 [21]. Therefore, the aim of the present study was to compare the effects of co-transplanting Fbs and SCs at a ratio of 1:2 to the effects of transplanting SCs or Fbs alone on peripheral nerve repair.

\section{Materials and Methods}

\section{Cell culture and detection in vitro}

Cell purification and enrichment

Normal culture medium (NCM) consisted of DMEM low-glucose medium (Thermo Fisher Scientific Life Sciences, Waltham, MA, USA), 10\% fetal bovine serum (FBS; Thermo Fisher), and penicillin-streptomycin (Sigma-Aldrich, St. Louis, MO, USA); Schwann cell culture medium (SCCM) consisted of NCM supplemented with basic fibroblast growth factor $(0.1 \mathrm{mg} / \mathrm{ml}$; Peprotech, Rocky Hill City, USA), heregulin- $\beta-10.1 \mathrm{mg} / \mathrm{ml}$ (Peprotech), and $2 \mu \mathrm{M}$ forskolin (Cayman, Ann Arbor, USA).

Male or female newborn (5-7 days old) wild-type C57BL/ 6 mice (wild-type mice; Shanghai SLAC Laboratory Animal Co. Ltd., Shanghai, China) were sacrificed by decapitation, and sciatic nerve (SN) segments were aseptically harvested from the mice under a dissecting microscope. Nerve segments were digested in an enzymatic solution containing collagenase NB4 (Serva, Heidelberg, Germany) and dispase II (neutral protease, grade II; Godo Shusei, Tokyo, Japan), both of which were dissolved in NCM at a concentration of $0.2 \%$. To digest the tissues, this enzymatic solution ( $50 \mu \mathrm{l}$ per segment) was added, and the tissues were then incubated at $37^{\circ} \mathrm{C}$ for $80 \mathrm{~min}$ in a cell incubator (Thermo Shandon, Cheshire, UK). The mixture was then centrifuged at $600 \times \mathrm{g}$ for 10 min. After the supernatant was removed, the cell pellet was resuspended in SCCM. The cells were cultured in a cell incubator in a humidified atmosphere of $5 \%$ carbon dioxide at $37^{\circ} \mathrm{C}$. After $48 \mathrm{~h}$, the culture medium was replaced with $0.2 \%$ dispase II diluted in NCM. After the mixture was incubated at $37^{\circ} \mathrm{C}$ for 20 mins, the cultured plates were shaken horizontally for 1-3 min to release detaching cells, and the suspended cells were then collected into a $15-\mathrm{ml}$ centrifuge tube (BD Falcon, USA) and centrifuged at $600 \times \mathrm{g}$ for $5 \mathrm{~min}$. After the supernatant was removed, the pellet was resuspended in SCCM. This SN-SC purification and enrichment procedure was repeated every $48 \mathrm{~h}$. After the first time the SN-SCs were removed from the culture plate using dispase, the adherent cells, which were mainly SN-Fbs, were passaged using trypsin-EDTA (Thermo Fisher). This purification and enrichment procedure was also repeated every $48 \mathrm{~h}$. SN-Fbs were mixed with SN-SCs at a 1:2 ratio (Fb\&SCs) after they were cultured for 6 days. They were then cultured for a further 2 days. Cells in all of the groups described below were passaged three times and cultured for a total of 8 days.

\section{Real-time quantitative reverse transcription-polymerase chain reaction}

The expression of neurotrophic genes, including nerve growth factor (NGF), brain-derived neurotrophic factor (BDNF), and glial-derived neurotrophic factor (GDNF), was analyzed in the three groups of cells (SN-SCs, SN-Fbs, Fb\&SCs) using reverse transcription-polymerase chain reaction (RT-PCR) after 8 days. The following primer sequences were used:

BDNF, F: 5'GGGTCACAGCGGCAGATAAA3' and R: 5'TGCAGCCTTCCTTGGTGTAAC3';

NGF, F: 5'GGAGCGCATCGAGTTTTGG3' and R: 5'CCTCACTGCGGCCAGTATAG3'; and GNDF, F: 5'CCGGTAAGAGGCTTCTCGAA3' and R: 5'TCAGTCTTTTAATGGTGGCTTGAA3'.

Total mRNA was extracted from the cultured cells using Trizol (Invitrogen, Carlsbad, CA, USA). The samples were quantified using RPL5 as a calibrator. Unknown samples were compared with the calibrator sample to obtain relative gene expression levels using the comparative CT method (2-meCT).

\section{Enzyme-linked immunosorbent assay}

Medium from each group was analyzed using enzyme-linked immunosorbent assay (ELISA) kits. A mouse NGF/NGF $\beta$ ELISA Kit (Syd Labs, Boston, 
USA) was used for NGF, a mouse BDNF ELISA Kit (Syd Labs) was used for BDNF, and a mouse GDNF ELISA Kit (Abnova, Taiwan, China) was used for GDNF according to the manufacturers' protocols. All samples were analyzed in triplicate, and absorbance was measured at $450 \mathrm{~nm}$ using a Varioskan Flash (Thermo Shandon).

\section{Animal experiments}

\section{Cell culture and preparation for transplantation}

All SN-SCs used for transplantation were derived from newborn (5-7 days old) male or female wild-type mice, and all SN-Fbs were derived from newborn (5-7 days old) male or female C57BL/6-Tg(CAG-EGFP)C14-Y01-FM131Osb mice (enhanced green fluorescent protein [EGFP] mice (Model Animal Research Center of Nanjing University, Nanjing, China) to facilitate post-surgical tracking. Cells cultured in vitro for a total of 8 days were centrifuged, resuspended in DMEM $\left(2 \times 10^{6}\right.$ cells:100 $\mu$ l DMEM), and then mixed with Matrigel (BD Bioscience, Franklin Lakes, NJ, USA) and stored on ice at $4^{\circ} \mathrm{C}$ overnight. They were then used in surgery at a ratio of 1:1. The mixture was aspirated into UltiCareTM insulin syringes (BD Bioscience) and incubated on ice during preparation for surgery.

\section{Experimental animals}

Male 6- to 8-week-old wild-type mice were used to establish animal models of 5 -mm SN defects. A total of 48 mice (22-24 g) were randomly divided into four groups ( $\mathrm{n}=12$ each) and submitted to the following treatments: transplantation with pure Matrigel (Control group), transplantation with pure $\mathrm{SN}-\mathrm{Fbs}$ (SN-Fb group), transplantation with $\mathrm{SN}-\mathrm{Fbs}$ mixed with SN-SCs at a 1:2 ratio (Fb\&SC group), and transplantation with pure SN-SCs (SN-SC group).

The mice were maintained under a 12-h light: 12-h dark cycle, fed standard mouse chow and provided water ad libitum. The mice were anesthetized prior to surgery using an intraperitoneal injection of sodium pentobarbital $(45 \mathrm{mg} / \mathrm{kg})$, and all subsequent surgical procedures were performed under aseptic conditions. All animal procedures were performed in accordance with the Institutional Animal Care guidelines of Shanghai First People's Hospital affiliated with Shanghai Jiao Tong University and ethically approved by the Ethics Committee of Shanghai First People's Hospital affiliated with Shanghai Jiao Tong University. The number of animals used was minimized, and animal suffering was avoided as much as possible.

\section{Surgical procedure}

All surgical procedures were performed under an optical microscope (DFC240C; Leica, Wetzlar, Germany) using microsurgical instruments (Zhangjiagang Oscar Medical Instruments Co., Ltd., Zhangjiagang, China). While each mouse was under anesthesia, a surgical incision (15 mm long) was made in the dorsal skin of the left hind limb, and the skin and hip muscle were then separated to expose the sciatic nerve. A 5-mm long segment of the sciatic nerve was excised, and $1 \mathrm{~mm}$ of each of the two resulting nerve stumps was inserted into each end of a 7-mm sterile PUR conduit (0.9-mm internal diameter, 1-mm external diameter) (Braun Medical Inc., Bethlehem, PA, USA) [22] [23]. The resulting 5-mm nerve defect was sutured into the lumen of the conduit using 10-0 fiber sutures (Shanghai Pudong Jinhuan Medical Products Co., Ltd., Shanghai, China) (Fig. 1A). A suspension of the appropriate cells $(3.2 \mu \mathrm{l}$ containing $3.2 \times 10^{4}$ cells) was then injected into the PUR conduit using a 29-gauge needle and an UltiCareTM insulin syringe ((BD Bioscience). The EGFP cells were visible after injection (Fig. 1B). The surgical incisions were then sutured closed using 5-0 nylon sutures (Shanghai Pudong Jinhuan Medical Products Co., Ltd.). All mice were treated postoperatively according to the guidelines of the Ethics Committee of Shanghai First People's Hospital affiliated with Shanghai Jiao Tong University [24].

\section{Electrophysiological examination}

Three months after surgery, six wild-type mice in each group were randomly selected to evaluate the activity of the affected gastrocnemius muscle using electromyography. After anesthesia was administered, an approximately 5-mm segment of the proximal end of the normal nerve was exposed. Double-hook stimulating electrodes were placed near the proximal ends of the normal nerves, reference electrodes were inserted into the axifugal part of the muscle, recording electrodes were inserted into the muscle belly of the middle segment of the gastrocnemius muscle, and grounding electrodes were inserted into the dorsal skin of the mouse. We used a Medtronic Keypoint electromyography machine (Medtronic Inc., Minneapolis, MN, USA) to record compound muscle action potentials (CMAPs) while gradually increasing current stimulation. The latency period and largest amplitude were analyzed.

\section{Histological assessment of regenerated nerves}

After the electrophysiological examination was performed, six mice in each group were perfused for nerve harvesting. The PUR conduits in these mice were then carefully removed to expose the regenerated nerves. All samples were fixed in a solution of $4 \%$ PFA in $10 \%$ sucrose for $2 \mathrm{~h}$ and then 
immersed in a $30 \%$ sucrose solution at $4^{\circ} \mathrm{C}$ for $24-48 \mathrm{~h}$. The nerve tissues were then embedded in optimal cutting temperature compound, cut into $10 \mu \mathrm{m}$-thick sections using a microtome (Shandon Finesse 325, Thermo Shandon), and processed for immunofluorescence or hematoxylin and eosin (HE) staining.

The following primary antibodies were used for immunofluorescence staining:

rabbit anti-neurofilament-H (NF-H; 1:100; Millipore, Billerica, MA, USA) to label axons, rabbit anti-myelin basic protein (MBP; 1:100; Abcam, Cambridge, UK) to label SCs, and rabbit anti-laminin (1:200; Abcam). The following secondary antibody was used: Alexa Fluor 594-conjugated donkey anti-rabbit IgG (1:500; Invitrogen). In addition, 4',6-diamidino-2phenylindole (DAPI; 1:500, Invitrogen) was used for nuclear staining to facilitate cell identification.

Tissue sections were permeabilized in phosphate-buffered saline (PBS, $\mathrm{pH}$ 7.4) containing $0.3 \%$ Triton $\mathrm{X}-100$, blocked in $10 \%$ goat serum in PBS $\left(37^{\circ} \mathrm{C}\right.$ for $\left.30 \mathrm{~min}\right)$, and incubated overnight with primary antibodies at $4^{\circ} \mathrm{C}$. All of these procedures were performed in a humidified incubator. After the nerve-tissue sections were incubated overnight, they were rinsed in PBS for $15 \mathrm{~min}$ and then incubated with secondary antibodies at $37^{\circ} \mathrm{C}$ for $45 \mathrm{~min}$. After nuclear staining was applied using DAPI, all of the sections were photographed using a TCS SP5 confocal laser-scanning microscope (Leica) and then stained using HE. The complete quantity of cross-sections through the middle segment of the regenerated axons was calculated according to NF immunofluorescent staining using IPP version 6.0 software (Media Cybernetics, Bethesda, MD, USA; $\mathrm{n}=6$ for each group).

The remaining six mice in each group were collected for toluidine blue staining and transmission electron microscope. After anesthesia, the PUR conduits were carefully removed, and the middle segment of the regenerated nerve was resected. The samples were fixed in 2\% glutaraldehyde in PBS at $4^{\circ} \mathrm{C}$ for $2 \mathrm{~h}$, rinsed, fixed with $1 \%$ osmic acid in PBS for $2 \mathrm{~h}$, and dehydrated through a graded ethanol series. The samples were then embedded in epoxy resin and cut into $1 \mathrm{~mm}$-thick sections using an LKB-V ultramicrotome (LKB Produkter AB, Ltd., Stockholm, Sweden). The sections were stained with $1 \%$ toluidine blue and then photographed using an Axiocam digital camera (Zeiss, Oberkochen, Germany). The 1 mm-thick epoxy resin-embedded nerve tissues were then cut into 50-nm ultrathin sections, stained with lead citrate, and photographed using a transmission electron microscope (H7650, Hitachi, Tokyo, Japan). Myelin sheath thickness and myelinated nerve fiber diameters were determined using 3000x images.

\section{Wet-weight analysis and Masson's trichrome staining of gastrocnemius muscles}

The nerve samples were removed, and the gastrocnemius muscles were then rapidly resected in both lower extremities and weighed using a precision electronic balance (JA1003N; Shanghai High-Precision Instruments Co., Ltd., Shanghai, China). A sample of the muscle belly of the central segment of the gastrocnemius muscle was collected and fixed in PFA at $4^{\circ} \mathrm{C}$ overnight. The samples were then dehydrated through a graded ethanol series, cleared in xylene, embedded in paraffin, and cut into $5 \mu \mathrm{m}$-thick sections using a Shandon Finesse 325 manual rotary microtome (Thermo Shandon). They were then stained using Masson's trichrome and analyzed under an E600 microscope (Nikon, Tokyo, Japan). The ratio of muscle fiber to collagen areas and muscle-fiber diameter were then calculated using IPP [24].
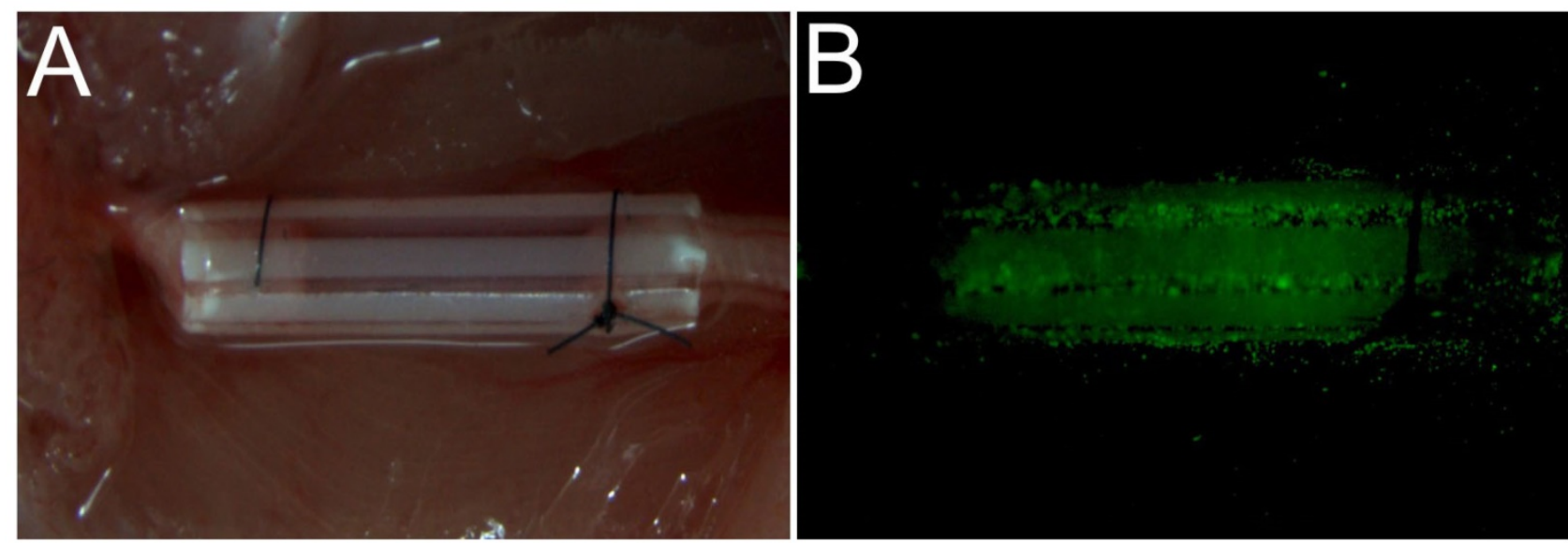

Figure 1. The surgical operation used to transplant different cell types in nerve defects. (A) A PUR catheter was used to bridge between the two stumps of the nerve defect. Different cell types were embedded in Matrigel and injected into a catheter. (B) Following injection, EGFP-expressing cells were visible on fluorescence microscopy. 


\section{Statistical analysis}

All statistical analyses were performed using SPSS version 19.0 (SPSS Inc., Chicago, IL, USA), and all data are expressed as the mean \pm standard deviation. All data were analyzed using one-way analysis of variance (ANOVA). Multiple comparisons between the groups were performed using the S-N-K method. A P value $<0.05$ was considered statistically significant.

\section{Results}

\section{Morphology and purification of SN-Fbs}

Cells obtained from the sciatic nerves of newborn mice were easily identified by their morphologies, as previously reported [25]. SN-Fbs were characterized by their polygonal shape and ovoid nuclei, which contain two or more nucleoli. In contrast, SN-SCs are much smaller and have a bipolar or tripolar cell morphology with phase-refractile properties. During purification, the volume of SN-Fbs rapidly increased, and the cytoplasm became gradually darker and flatter (Fig. 2A-D). In addition, the purity of the Fbs reached $95.36 \pm 0.29 \%$ after the third purification (Fig. 2E).

\section{Evaluation of PCR and ELISA results in vitro}

Neurotrophic factors play essential roles in the maintenance and survival of peripheral neuronal cells and are always implicated in nerve regeneration [26-28]. After 8 days, cells in all of the groups were analyzed using PCR, and their supernatants were assessed using ELISA. The trends observed in ELISA were similar trends to those observed in PCR analysis except for the expression of NGF in the SN-Fb group. Genes are regulated at multiple levels after transcription and before translation of the protein. These mechanisms include the regulation of translation, the modification or folding of proteins, and the degradation of mRNA. Thus, the specific mechanism that contributes to the difference between the mRNA and protein expression of NGF in the $\mathrm{SN}-\mathrm{Fb}$ group is likely to be very complex and requires further exploration.

NGF and BDNF were expressed at higher levels while GDNF was expressed at lower levels in the $\mathrm{SN}-\mathrm{Fb}$ group than in the SN-SC group, suggesting that $\mathrm{SN}-\mathrm{Fbs}$ may exert neurotrophic factor-mediated beneficial effects on nerve regeneration. However, NGF, BDNF and GDNF were expressed at significantly higher levels in the Fb\&SC group than in the SN-SC group, suggesting that combining SN-Fbs and SCs at a ratio of 1:2 promoted the production of neurotrophic factors and thereby contributed to nerve repair (Fig. 3).

\section{Macroscopic observation of regenerated nerves at $\mathbf{3}$ months and HE staining}

A macroscopic examination of the regenerated nerves showed that nerve continuity was fully recovered in all groups at 3 months after surgery (Fig. $4 \mathrm{~A}-\mathrm{D})$. In addition, the diameters of the regenerated nerves were thicker in the Fb\&SC and SN-SC groups than in the other groups (Fig. 4E-H).
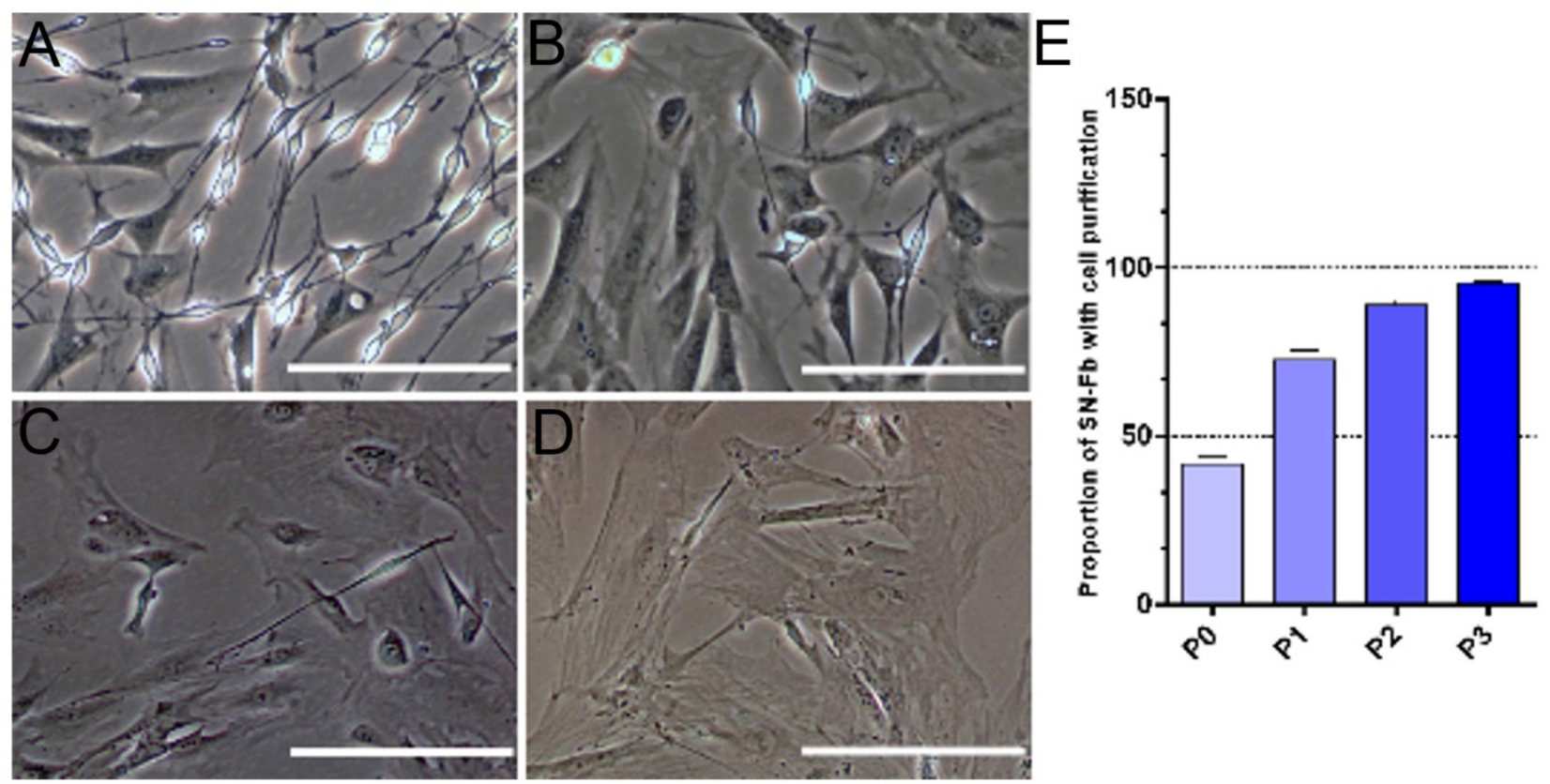

Figure 2. Purification of SN-Fbs obtained from primary cultures in vitro. (A) SN-Fbs and SN-SCs at 2 days after primary culture (PO). (B) SN-Fbs at 2 days after the first purification (P1). (C) SN-Fbs at 2 days after the second purification (P2). (D) SN-Fbs at 2 days after the third purification (P3). (E) Proportions of SN-Fbs at different stages. All data are expressed as the mean $\pm \operatorname{SD}(n=6$ in each group). Scale bar $=250 \mu \mathrm{m}$. 
A

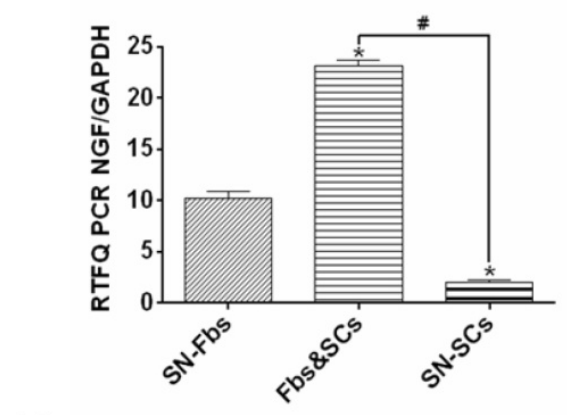

D

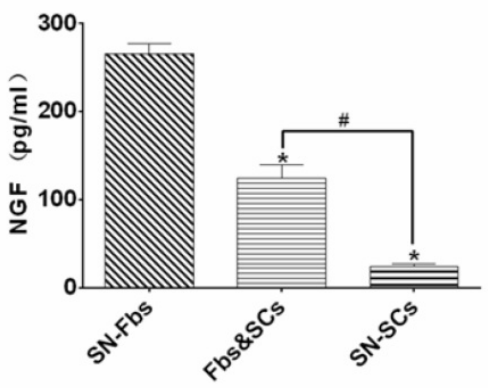

B

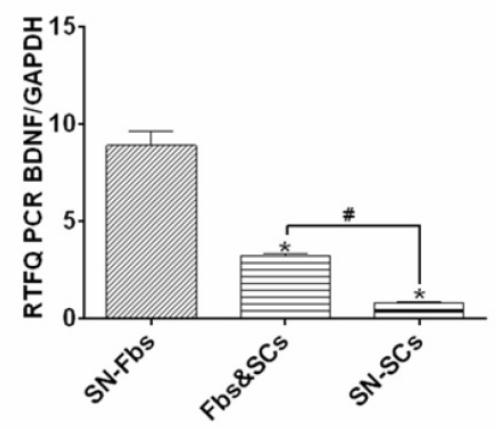

E

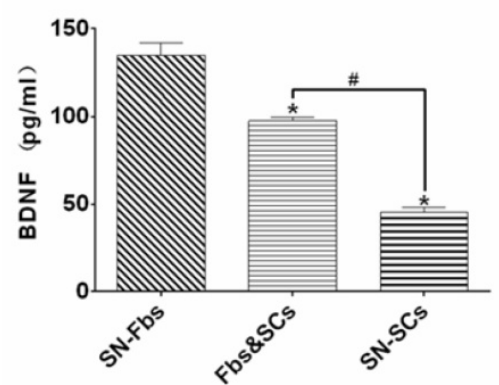

C

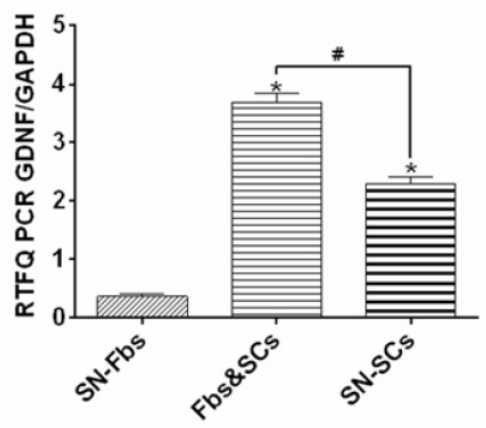

F

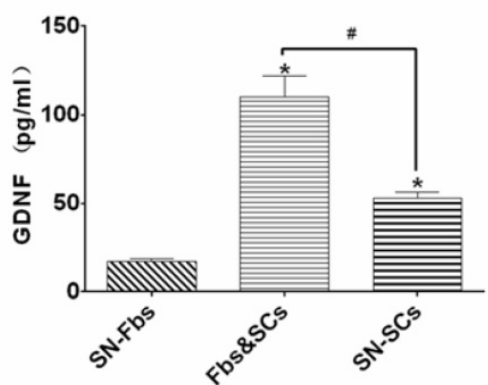

Figure 3. Detection of neurotrophic factors, including NGF, BDNF, and GDNF, at the mRNA (A-C) and protein (D-E) levels in all groups in vitro. $* P<0.05$ vs. the SN-Fb group. \# $P<0.05$ between the two groups. All data are expressed as the mean \pm SD ( $n=6$ in each group).
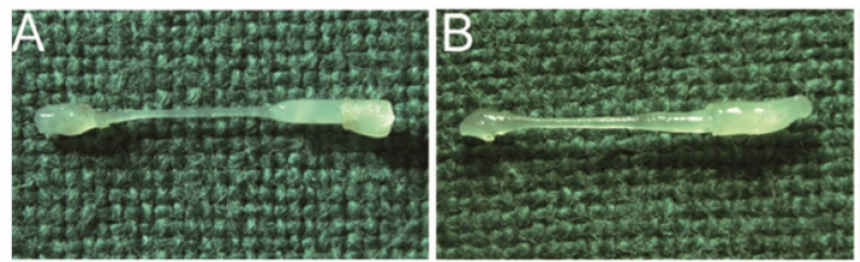

$\mathrm{E}$
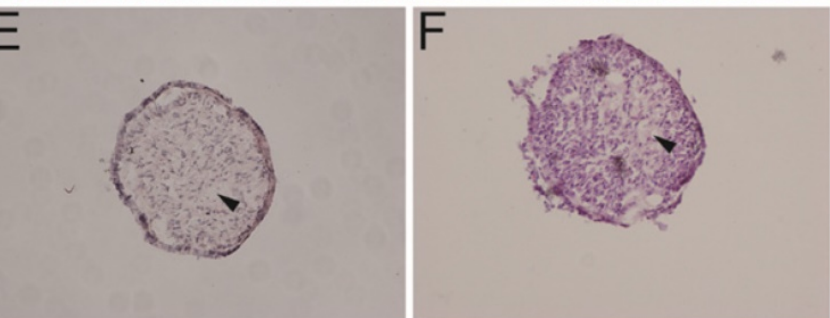
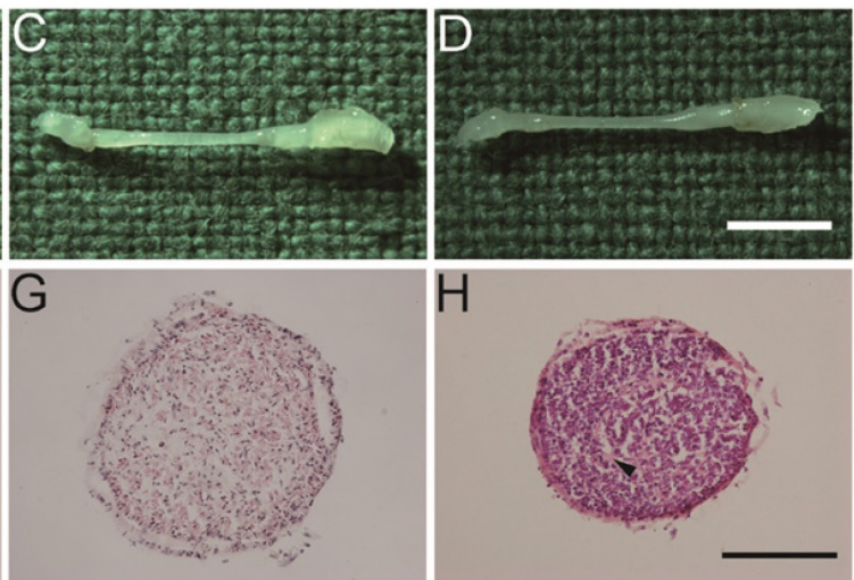

Figure 4. (A-D) Macroscopic view of regenerated nerves after 3 months. Scale bar $=3 \mathrm{~mm}$. (E-H) HE staining of transverse sections obtained from the middle segment of the regenerated nerves. Scale bar $=100 \mu \mathrm{m}$. (A, E) Control group. (B, F) SN-Fb group. (C, G) Fb\&SC group. (D, H) SN-SC group. Arrowheads showed area in subdued color without nuclei.

HE staining demonstrated that nuclei were normally distributed in the Fb\&SC group (Fig. 4G). However, differently sized areas that displayed subdued color and no nuclei were considered extracellular matrix (ECM) deposits, and these were randomly distributed in the Control, $\mathrm{SN}-\mathrm{Fb}$ and SN-SC groups (Fig. 4 E, F, H).

\section{Immunofluorescence staining for NF-H in regenerated axons}

To investigate the regenerated axons, we performed immunofluorescence staining for NF-H at three months after surgery. In regenerated nerves, EGFP-positive SN-Fbs presented linear, semicircular or circular features wrapping NF-H-positive axons and MBP-positive SCs in the SN-Fb (Fig. 5B, 6B) and Fb\&SC (Fig. 5C, 6C) groups. This labeling coincided with perineurium-like structures that were considered the fascicles of the regenerated nerves [29, 30]. 

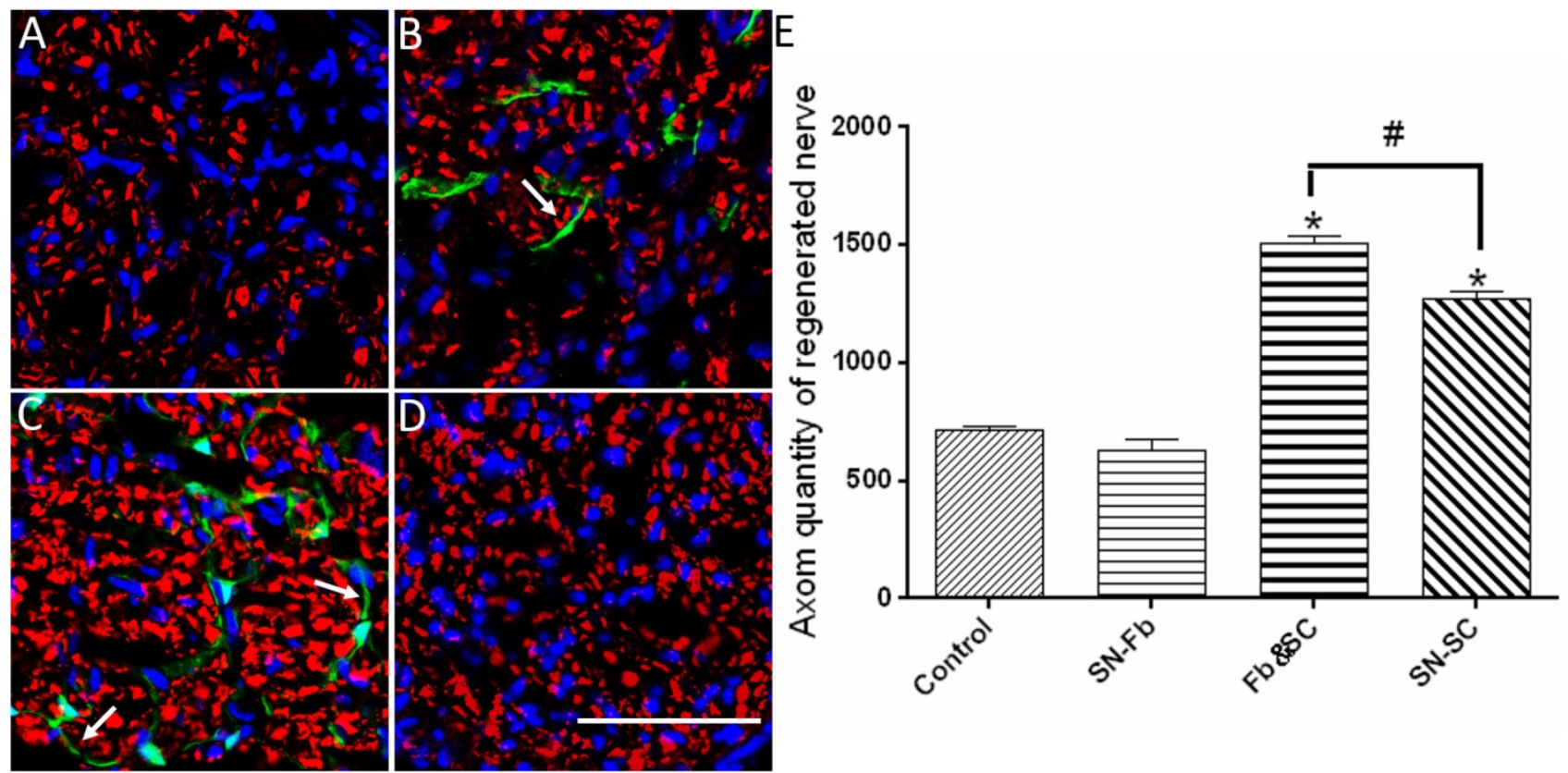

Figure 5. Immunofluorescence staining of axons in transverse sections from the middle segment of regenerated nerves with NF-H (red). (A) Control group. (B) SN-Fb group. (C) Fb\&SC group. (D) SN-SC group. (E) Statistical analysis of axon quantity in the regenerated nerve. All data are expressed as the mean \pm SD ( $\mathrm{n}=6$ in each group). $* P<0.05$ vs. the Control group, \# $P<0.05$ between the two groups. Transplanted SN-Fbs appear green (B, C and $E$ with arrow). Scale bar $=50 \mu \mathrm{m}$.

We next calculated the number of regenerated axons in each group. Remarkably, we found that the number of regenerated axons was significantly higher in the $\mathrm{Fb} \& \mathrm{SC}$ group than in the SN-SC, SN-Fb and Control groups (Fig. 5E). There was no significant difference in the number of regenerated axons between the $\mathrm{SN}-\mathrm{Fb}$ and Control groups (Fig. 5E).

These data suggest that SCs play an important role in nerve restoration, as previously demonstrated [31]. Moreover, combining SN-Fbs with SCs resulted in an optimal effect, whereas SN-Fbs alone had no effect on regeneration.

\section{Immunofluorescence staining for MBP in regenerated SCs}

To evaluate the role of SN-Fbs in myelination, we performed immunofluorescence staining for MBP and used the ratio of the area that was positively stained for MBP to the complete cross-sectional area as an assessment. The results showed that the MBP positive area was smaller in the $\mathrm{SN}-\mathrm{Fb}$ group than in the SN-SC group but similar to the Control group (Fig. 6). Surprisingly, the ratio was higher in the $\mathrm{Fb}$ \&SC group than in other groups (Fig. 6E), indicating that transplanting $\mathrm{SN}-\mathrm{Fbs}$ and $\mathrm{SCs}$ at a 1:2 ratio provided a beneficial microenvironment that effectively promoted the myelination of SCs, whereas transplanting $\mathrm{SN}-\mathrm{Fb}$ alone did not.

\section{Toluidine blue staining and ultrastructural analysis in electron microscopy}

To evaluate regeneration efficacy, we analyzed semi-thin transverse sections that were stained with toluidine blue. A large number of regenerated myelinated nerve fibers were distributed in the middle segment of the nerve sample in all of the groups (Fig. 7A-D). Myelinated nerve fibers had clearly larger diameters in the Fb\&SC group than in the other groups, but there was no significant difference between the $\mathrm{SN}-\mathrm{Fb}$ and Control groups. These trends were identical to those observed in the statistical analysis of myelinated nerve diameter performed using electron microscopy (Fig. 7G).

Transmission electron microscopy was used to observe the ultrastructural structures of regenerated nerves. Perineurium or fascicles with different sizes, each consisting of several layers of fibroblasts (arrows), encircled SCs and axons in all of the groups (Fig. 7E, F, H, I). Similar results are also shown in Fig. 7A-D.

Myelin sheath thickness was calculated to evaluate the level of myelination. Myelin sheath thickness was significantly greater in the Fb\&SC group than in the SN-SC group. While the thicknesses of the $\mathrm{SN}-\mathrm{Fb}$ and Control groups were similar, they were distinctly less than those observed in the other two groups (Fig. 7J). 

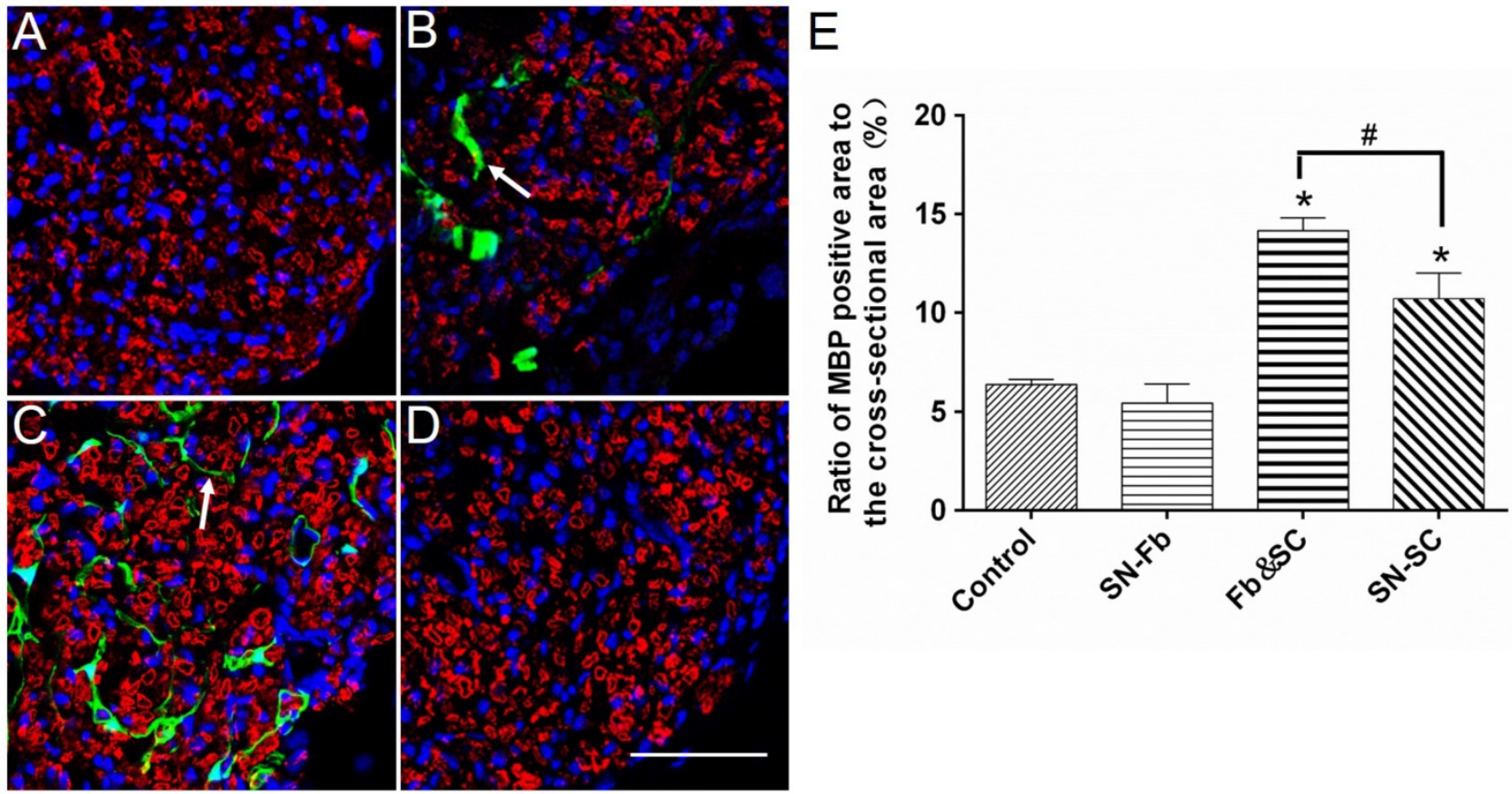

Figure 6. Immunofluorescence staining for MBP (red) in transverse sections obtained from the middle segments of regenerated nerves against myelinated Schwann cells. (A) Control group. (B) SN-Fb group. (C) Fb\&SC group. (D) SN-SC group. (E) Statistical analysis of MBP expression. All data are expressed as the mean \pm SD ( $n=6$ in each group). $* \mathrm{P}<0.05$ vs. the Control group, \# $\mathrm{P}<0.05$ between the two groups. Transplanted SN-Fbs appear green $(B, C$ and $E$ with arrow). Scale bar $=$ $50 \mu \mathrm{m}$.

\section{Evaluation of laminin in regenerated nerves}

In the ECM, laminin presented an even, grid-like distribution in the Control group but an excessive, uneven, and aggregated distribution in the $\mathrm{SN}-\mathrm{Fb}$ group (Fig. 8B). These data demonstrate that transplanting SN-Fbs stimulated the production of laminin-positive ECM. We propose that the excessive, uneven, and aggregated distribution of this protein in the ECM probably occupied what would otherwise have been space for the regenerated nerve, thus preventing nerve regeneration, consistent with previous reports [32]. Interestingly, the lattices observed in the Fb\&SC group (Fig. 8C) were more homogeneous than those observed in the SN-SC group, but there was some aggregated ECM in the SN-SC group (Fig. 8D). These results indicate that applying an optimal ratio of Fbs and SCs improves how favorable the microenvironment is for nerve regeneration.

\section{Evaluation of amyotrophy}

Macroscopic views of affected gastrocnemius muscles obtained at 3 months after surgery are shown in Fig. 9A-D. Similar degrees of atrophy were observed in the Control and SN-Fb groups, which showed remarkably higher levels of atrophy than were observed in the Fb\&SC and SN-SC groups. The wet weight of the gastrocnemius muscle in the Fb\&SC group was statistically higher than that in the other three groups (Fig. 9I).
Masson's trichrome staining was used to evaluate muscle-fiber atrophy and collagen sedimentation. There were no significant differences between the $\mathrm{SN}-\mathrm{Fb}$ and Control groups in muscle fiber atrophy and collagen sedimentation, but both were much more severe in these two groups than in the Fb\&SC and SN-SC groups (Fig. 9E-H). In addition, less amyotrophy and collagen sedimentation were observed in the Fb\&SC group than in the SN-SC group (Fig. 9J, K).

\section{Electrophysiology}

Electromyography was performed to evaluate conduction from regenerated nerves to the gastrocnemius muscle (Fig. 10). The CMAP peak and latency periods were calculated and their significance analyzed. Compared with the Control group, preferable recovery indexes were observed in Fb\&SC and SN-SC groups, but the improvement was far more dramatic in the Fb\&SC group. In addition, there were no significant differences between the $\mathrm{SN}-\mathrm{Fb}$ and Control groups.

\section{Discussion}

In this study, we explored the effects of co-transplanting $\mathrm{SN}-\mathrm{Fbs}$ with $\mathrm{SCs}$ on nerve regeneration. The main finding of our study is that co-transplanting SN-Fbs with $\mathrm{SCs}$ at a 1:2 ratio conferred good regenerative effects and resulted in functional recovery at 3 months after surgery. 
The histological structure of regenerated nerves was grossly different from that of normal peripheral nerves. In normal nerves, the perineurium consists of six to eight layers of compacted, concentric, flattened cells and has a characteristic organization that includes tight junctional arrays that contribute to the blood-nerve barrier [30]. In contrast, in all groups in this study, the interiors of regenerated nerves were divided into fascicles that were made of one to six unwound, flattened layers (Fig. 7). The appearance of these fascicles is consistent with the general construction of regenerated nerves observed in previous studies $[29,30,33]$. Unlike what is observed in a normal perineurium, the fascicles in the regenerated nerves were connected by leaky junctions that allowed both proteins and inflammatory cells to enter [30], which is necessary during the early recovery period. Whether these fascicles eventually form a normal perineurium requires further study. Moreover, EGFP-positive SN-Fbs were identified in the fascicles of the SN-Fb and Fb\&SC groups (Fig. 5, 6). However, some of these cells had linear and semicircular rather than circular features, and this indicates that endogenous Fbs, which were not green, and EGFP-positive SN-Fbs participated in a collaborative manner during the construction of these fascicles.
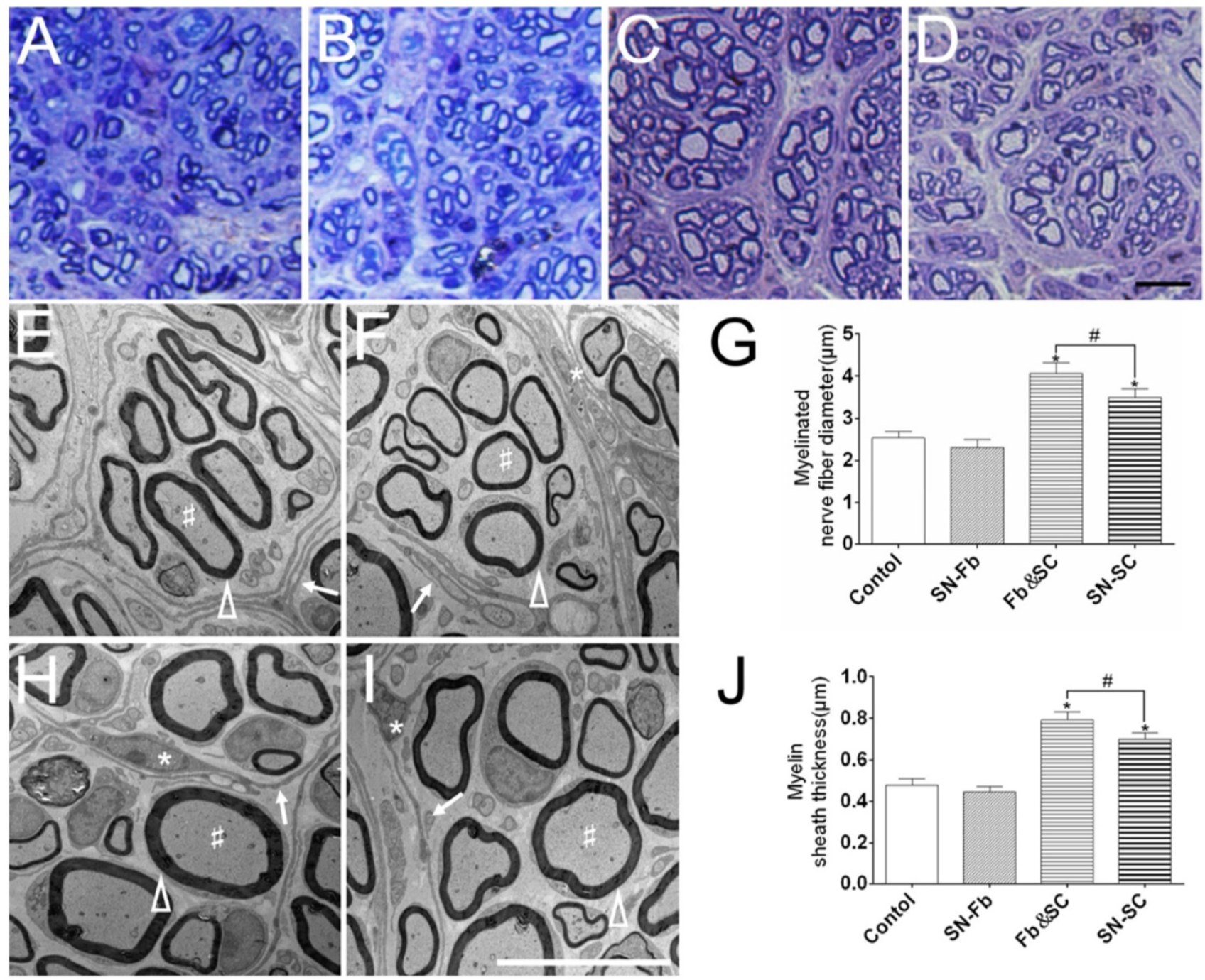

Figure 7. (A-D) Toluidine blue staining of semi-thin sections obtained from the middle segment of regenerated nerves 3 months after surgery. Scale bar $=20 \mu \mathrm{m}$. $(E$, $\mathrm{F}, \mathrm{H}, \mathrm{I})$ An ultrastructural analysis was performed using electron microscope to evaluate the regenerated axons and remyelination in each group. Scale bar $=10 \mu \mathrm{m}$. (A, E) Control group. (B, F) SN-Fb group. (C, H) Fb\&SC group. (D, G) SN-SC group. White triangle, myelin sheath; arrow, perineurium; star, cell nuclei of fibroblasts; pound sign, myelinated nerve fibers. Myelinated nerve fiber diameter $(G)$ and myelin sheath thickness $(J)$ were quantitatively evaluated and statistically analyzed. All data are expressed as the mean $\pm S D$ ( $n=6$ in each group). $* P<0.05$ vs. the Control group, $\# P<0.05$ between the two groups. 

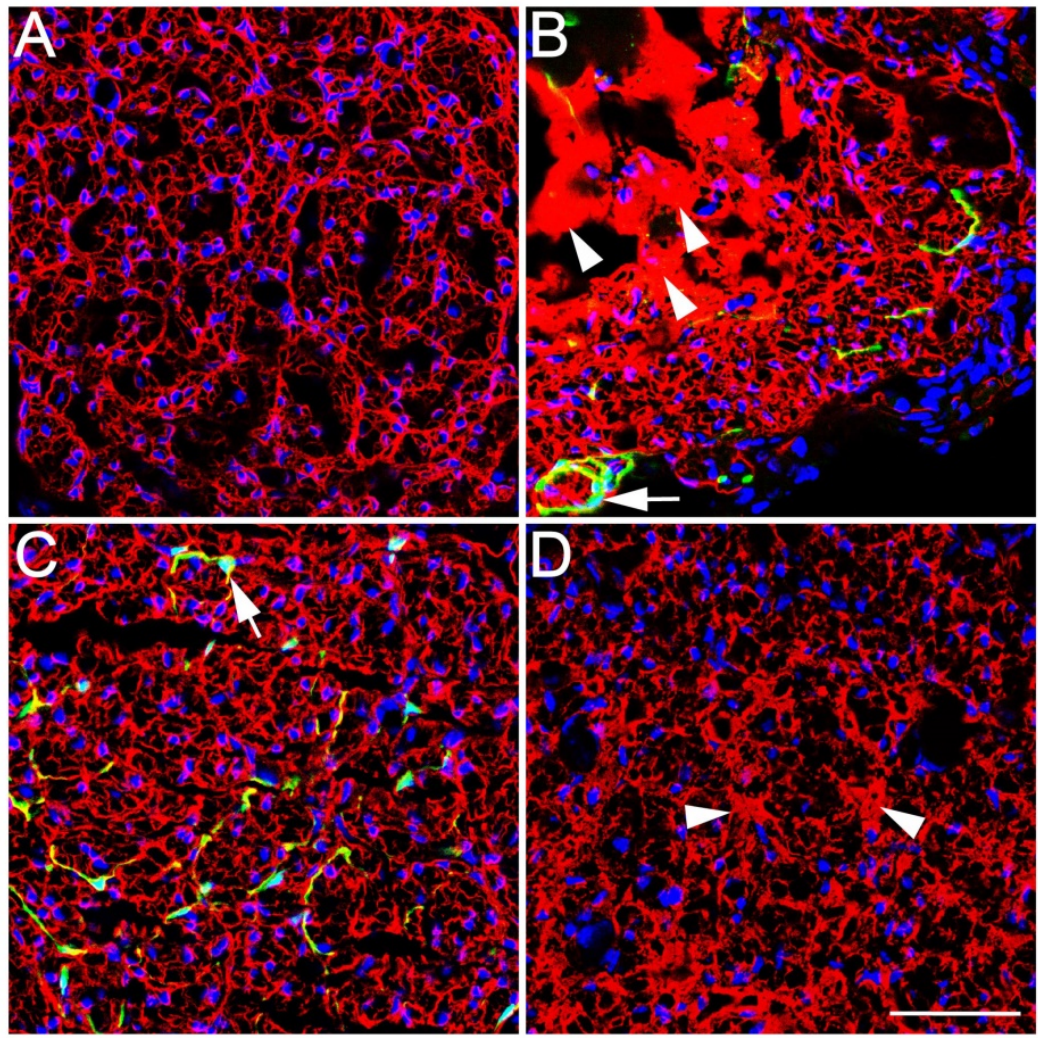

Figure 8. Immunofluorescence staining for laminin ( $\mathrm{LN}$, red) in transverse sections obtained from the middle segment of regenerated nerves. (A) Control group. (B) $\mathrm{SN}-\mathrm{Fb}$ group. (C) Fb\&SC group. (D) SN-SC group. Scale bar $=50 \mu \mathrm{m}$. Transplanted SN-Fbs appear green (B and C with arrow), the arrow head in B and D indicate an uneven and aggregated distribution.
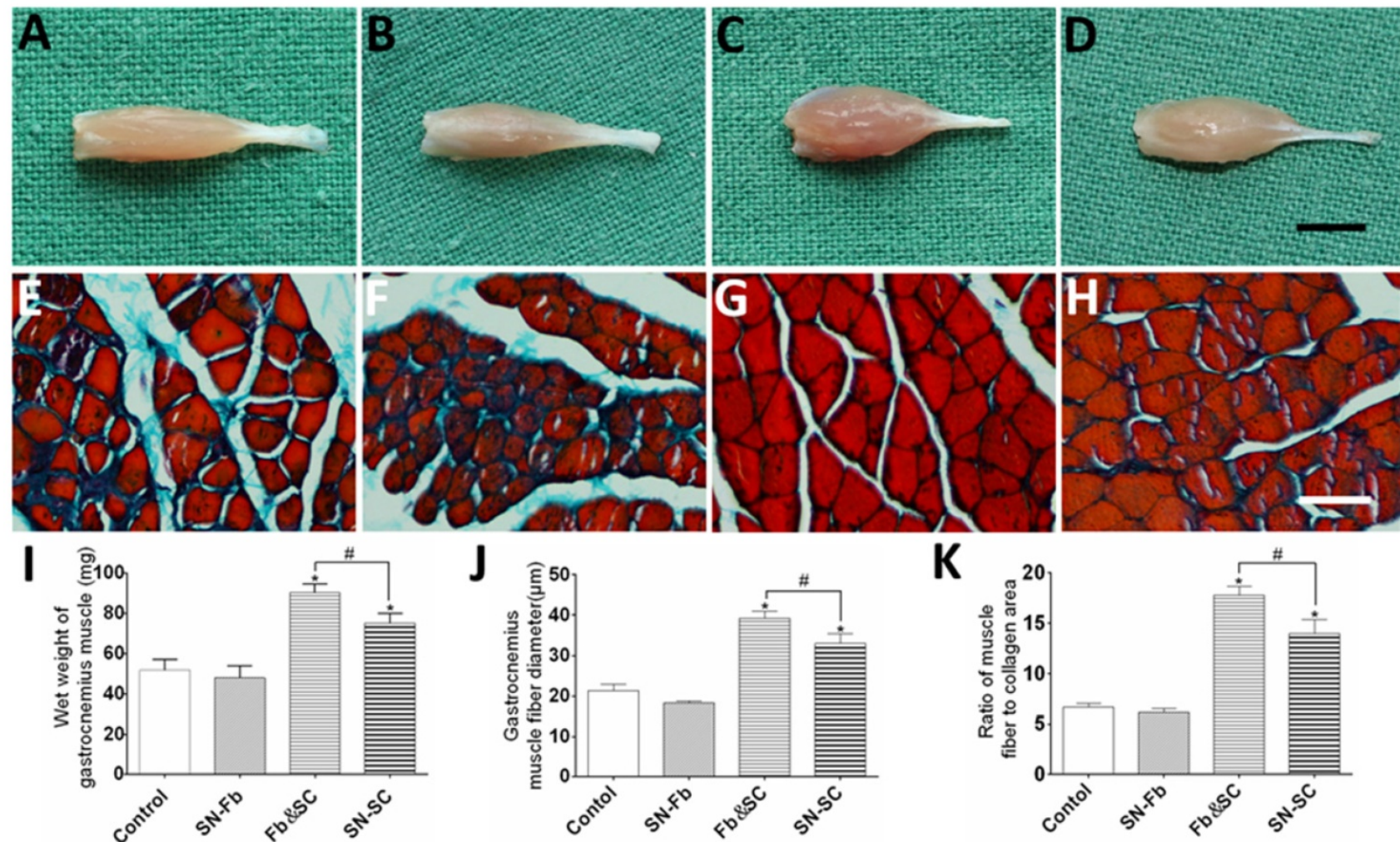

Figure 9. (A-D) Macroscopic views of gastrocnemius muscles at three months after surgery. Scale bar $=5 \mathrm{~mm}$. (E-H) Masson's trichrome staining of transverse sections obtained from the middle segment of the gastrocnemius muscle. Scale bar $=50 \mu m$. (I) wet weight of the gastrocnemius muscles. (J, K) Analysis of gastrocnemius muscle fiber diameter and the ratio of muscle fiber area to collagen area according to Masson's trichrome staining. (A, E) Control group. (B, $\mathrm{F}$ ) $\mathrm{SN}$-Fb group. (C, G) Fb\&SC group. (D, H) SN-SC group. All data are expressed as the mean $\pm S D(n=6$ in each group). *P< 0.05 vs. the Control group, \# $P<0.05$ between the two groups. 

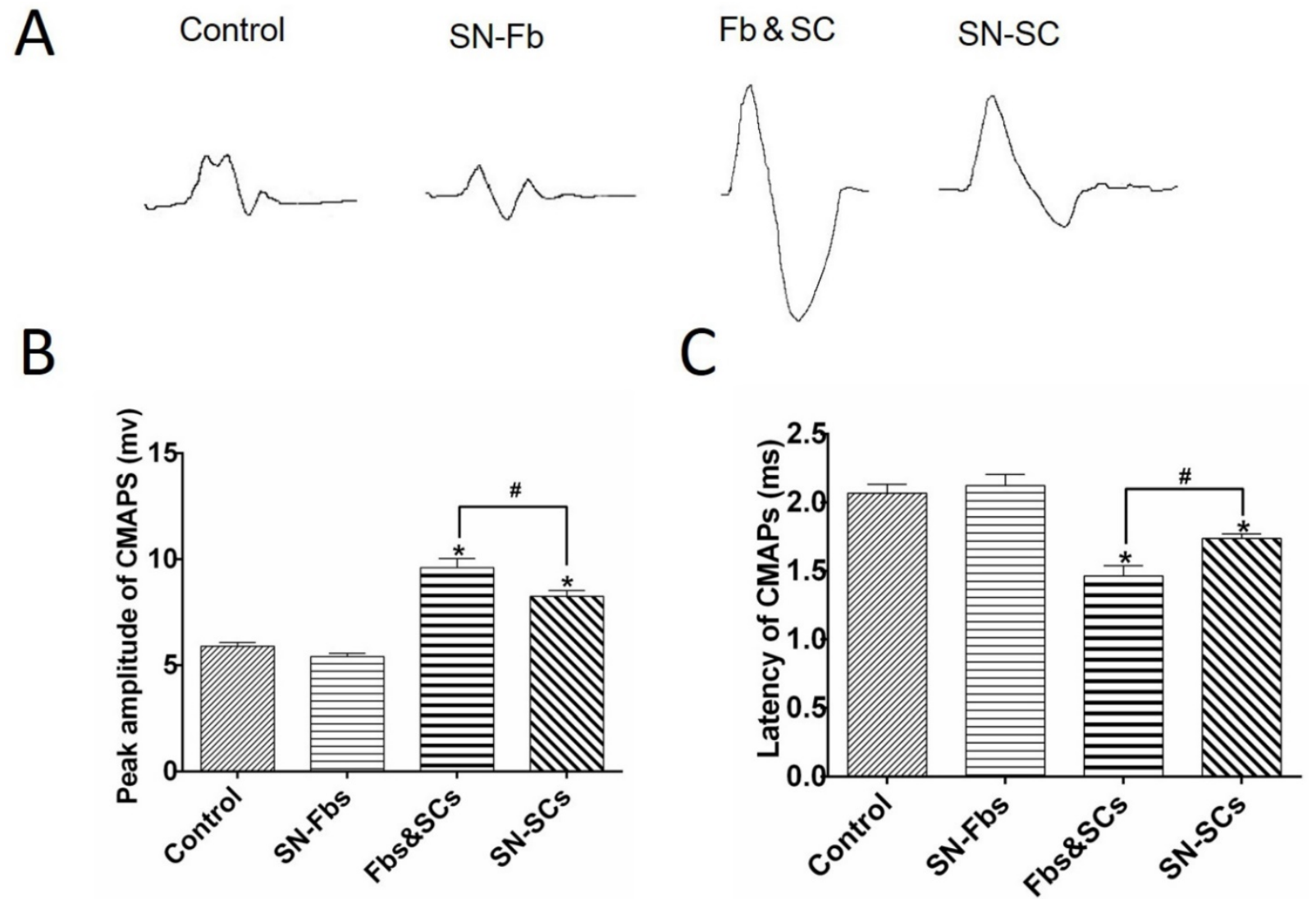

Figure 10. Electrophysiological evaluation. (A) Electromyography of the gastrocnemius muscle in each group at three months after surgery. The peaks (B) and latencies $(C)$ of the action potentials were recorded and statistically analyzed. All data are expressed as the mean $\pm S D(n=6$ in each group).*P< 0.05 vs. the Control group, \# $P<0.05$ between the two groups.

Recent studies have demonstrated that fibroblasts originated from different tissues display different patterns of gene expression and have functions that are specifically beneficial to their tissues [16]. Other studies have shown that these local functions can be maintained in cultured cells in vitro using HOX genes [34]. Similarly, a large number of in vitro studies have demonstrated that SN-Fbs increase the migration of SCs and the ensheathment of axons $[9,10]$. In addition, our results show that SN-Fbs cultured in vitro secrete large amounts of a variety of beneficial neurotrophic factors (Fig. 3). However, a minority of researchers have argued that suppressing fibroblasts reduces scar formation and increases the regeneration of axons [35]. In this study, when cells were transplanted into a nerve defect, there was no significant difference between the $\mathrm{SN}-\mathrm{Fb}$ and Control groups, indicating that $\mathrm{SN}-\mathrm{Fbs}$ did not hinder nerve regeneration. However, while they were capable of secreting neurotrophic factors, they failed to facilitate nerve regeneration, possibly because of excessive ECM aggregation (Fig. 8). Fbs play a critical role in wound healing by producing ECM and generating contractile forces [36] in their host tissues. Similarly, $\mathrm{SN}-\mathrm{Fbs}$ are the first cells to bridge across a wound, and they act by secreting ECM [11] and guiding the directional migration of SCs and axons from nerve stumps [12]. However, excessive ECM aggregation has been shown to hinder nerve regeneration [32].

In terms of historical method of evaluating results (i.e., MBP immunofluorescent staining, toluidine blue staining, and ultrastructural observations made using electron microscopy), the richest and ripest myelination was observed in the Fb\&SC group rather than the SN-SC group. According to previous reports, BDNF stimulates myelination by activating P75NTR in axons and SCs [37-39]. This is consistent, to some extent, with the results of our study. In fact, in cells cultured in vitro, much higher levels of BDNF were detected in the Fb\&SC group than in the SN-SC group. However, BDNF secretion was highest in the $\mathrm{SN}-\mathrm{Fb}$ group, while MBP-positivity was not. We hypothesize that these cells produce too much ECM, thus inhibiting SC proliferation. The mechanism involved in the acceleration of myelination is complex and involves multiple processes, and further studies are therefore needed to clarify them.

The light areas with no nuclei observed in the $\mathrm{SN}-\mathrm{Fb}$ and SN-SC groups indicate the accumulation 
of bulk-shaped ECM. No clear massive deposits of ECM were observed in the Fb\&SC group (Fig. 4, 8), demonstrating that in this group, SN-Fbs probably collaborate with SCs to regulate the production and accumulation of ECM. A moderate amount of ECM, and especially the protein laminin, is beneficial to neuronal survival and axon regeneration. However, excessive ECM aggregation can potentially occupy the regenerated space, which can prevent axons and SCs from getting through, which may hinder regeneration. It has been suggested that SN-SCs are the optimal cell type for repairing peripheral nerve defects $[12,31]$, but several lesions occupied by ECM were detected in the SN-SC group, consistent with previous reports [40]. We conclude from these data that this may be one of the reasons that the Fb\&SC group exhibited an advantage over the SN-SC group in terms of nerve repair.

In short, treatment with Fb\&SC resulted in optimal conditions for nerve regeneration. The number of regenerated axons, the mean diameter of the regenerated axons, and the thickness of the myelin sheath on these axons were all significantly different from the corresponding values in SN-SC group. Additionally, Masson's trichrome staining and electrophysiological analyses are widely accepted techniques for functionally evaluating sciatic nerve repair $[41,42]$. The results obtained in the Fb\&SC group were preferable to those obtained in the SN-SC group with regard for functional recovery. The capacity of Fbs to communicate with other cells in different tissues makes them a central component of tissue biology [43]. Similar to previous reports [16] showing that reciprocal interactions between Fbs and their corresponding epithelial cells play critical roles in augmenting tissue repair and remodeling, the results of this study demonstrate that co-transplanting Fbs with SCs has a preferable advantage over transplanting SCs alone in $5 \mathrm{~mm}$ nerve defects in mice.

Nerve regeneration is a complex procedure that involves the precise coordination of and dynamic interactions between a variety of cells and factors. The current study supports the notion that when SN-Fbs and SCs are transplanted at a ratio of 1:2, they cooperate to better support nerve regeneration. This approach may also have broader implications. For example, these cell combinations could be combined with biomaterials, such as scaffolding, in nerve tissue engineering.

\section{Conclusions}

Transplanting SN-Fbs together with SCs at a ratio of 1:2 produced superior results when compared to SCs transplanted alone in nerve regeneration and functional recovery. This effect was mainly attributed to the more abundant production of neurotrophic factors and the appropriate production and distribution of ECM.

\section{Acknowledgements}

This work was financially supported by the National Natural Science Foundation of China (31170932).

\section{Author Contributions}

Y.J., Z.S. and Y.W. designed the study; Y.W., G.W., L.C. and J.C. performed the experiments; Z.L. and Z.Z. provided experimental technical support, and Y.W., D.L. and H.S. wrote the paper.

\section{Competing Interests}

The authors have declared that no competing interest exists.

\section{References}

[1] Siemionow M, Bozkurt M, Zor F. Regeneration and repair of peripheral nerves with different biomaterials: Review. MICROSURG. 2010; 30:574-88.

[2] Xu Y, Zhang Z, Chen X, et al. A Silk Fibroin/Collagen Nerve Scaffold Seeded with a Co-Culture of Schwann Cells and Adipose-Derived Stem Cells for Sciatic Nerve Regeneration. PLOS ONE. 2016; 11:e147184.

[3] Kouyoumdjian JA. Peripheral nerve injuries: a retrospective survey of 456 cases. MUSCLE NERVE. 2006; 34:785-8.

[4] Fan L, Yu Z, Li J, et al. Schwann-like cells seeded in acellular nerve grafts improve nerve regeneration. BMC Musculoskelet Disord. 2014; 15:165.

[5] Pan M, Wang X, Chen $Y$, et al. Tissue engineering with peripheral blood-derived mesenchymal stem cells promotes the regeneration of injured peripheral nerves. EXP NEUROL. 2017; 292:92-101.

[6] Wang Y, Zhao Y, Sun C, et al. Chitosan Degradation Products Promote Nerve Regeneration by Stimulating Schwann Cell Proliferation via miR-27a/FOXO1 Axis. MOL NEUROBIOL. 2016; 53:28-39.

[7] $\mathrm{Hu} \mathrm{Y}, \mathrm{Wu} \mathrm{Y,} \mathrm{Gou} \mathrm{Z,} \mathrm{et} \mathrm{al.} \mathrm{3D-engineering} \mathrm{of} \mathrm{Cellularized} \mathrm{Conduits} \mathrm{for}$ Peripheral Nerve Regeneration. Sci Rep. 2016; 6:32184.

[8] Joseph NM, Mukouyama YS, Mosher JT, et al. Neural crest stem cells undergo multilineage differentiation in developing peripheral nerves to generate endoneurial fibroblasts in addition to Schwann cells. DEVELOPMENT. 2004; 131:5599-612.

[9] Dreesmann L, Mittnacht U, Lietz M, et al. Nerve fibroblast impact on Schwann cell behavior. EUR J CELL BIOL. 2009; 88:285-300.

[10] van Neerven SG, Pannaye P, Bozkurt A, et al. Schwann cell migration and neurite outgrowth are influenced by media conditioned by epineurial fibroblasts. NEUROSCIENCE. 2013; 252:144-53.

[11] Zhang Z, Yu B, Gu Y, et al. Fibroblast-derived tenascin-C promotes Schwann cell migration through beta1-integrin dependent pathway during peripheral nerve regeneration. GLIA. 2016; 64:374-85.

[12] Parrinello S, Napoli I, Ribeiro S, et al. EphB signaling directs peripheral nerve regeneration through Sox2-dependent Schwann cell sorting. CELL. 2010; 143:145-55.

[13] Obremski VJ, Johnson MI, Bunge MB. Fibroblasts are required for Schwann cell basal lamina deposition and ensheathment of unmyelinated sympathetic neurites in culture. J Neurocytol. 1993; 22:102-17.

[14] Obremski VJ, Wood PM, Bunge MB. Fibroblasts promote Schwann cell basal lamina deposition and elongation in the absence of neurons in culture. DEV BIOL. 1993; 160:119-34.

[15] Kuperwasser C, Chavarria T, Wu M, et al. Reconstruction of functionally normal and malignant human breast tissues in mice. Proc Natl Acad Sci U S A. 2004; 101:4966-71.

[16] Wong T, McGrath JA, Navsaria $\mathrm{H}$. The role of fibroblasts in tissue engineering and regeneration. Br J Dermatol. 2007; 156:1149-55.

[17] Werner S, Krieg T, Smola H. Keratinocyte-fibroblast interactions in wound healing. J INVEST DERMATOL. 2007; 127:998-1008.

[18] Aoki S, Toda S, Ando T, et al. Bone marrow stromal cells, preadipocytes, and dermal fibroblasts promote epidermal regeneration in their distinctive fashions. MOL BIOL CELL. 2004; 15:4647-57.

[19] El-Ghalbzouri A, Gibbs S, Lamme E, et al. Effect of fibroblasts on epidermal regeneration. Br J Dermatol. 2002; 147:230-43.

[20] Fimiani M, Pianigiani E, Di Simplicio FC, et al. Other uses of homologous skin grafts and skin bank bioproducts. CLIN DERMATOL. 2005; 23:396-402. 
[21] Schubert T, Friede RL. The role of endoneurial fibroblasts in myelin degradation. J Neuropathol Exp Neurol. 1981; 40:134-54.

[22] Cui T, Yan Y, Zhang R, et al. Rapid prototyping of a double-layer polyurethane-collagen conduit for peripheral nerve regeneration. Tissue Eng Part C Methods. 2009; 15:1-9.

[23] Soldani G, Varelli G, Minnocci A, et al. Manufacturing and microscopical characterisation of polyurethane nerve guidance channel featuring a highly smooth internal surface. BIOMATERIALS. 1998; 19:1919-24.

[24] Yang XN, Jin YQ, Bi H, et al. Peripheral nerve repair with epimysium conduit. BIOMATERIALS. 2013; 34:5606-16.

[25] $\mathrm{Wu} W$, Jin YQ, Kretlow JD, et al. Purification of Schwann cells from adult rats by differential detachment. BIOTECHNOL LETT. 2009; 31:1703-8.

[26] Lewin GR, Carter BD. Neurotrophic factors. Berlin Heidelberg, Germany; Springer-Verlag, 2014

[27] Terenghi G. Peripheral nerve regeneration and neurotrophic factors. J ANAT. 1999; 194 ( Pt 1):1-14.

[28] Acheson A, Barker PA, Alderson RF, et al. Detection of brain-derived neurotrophic factor-like activity in fibroblasts and Schwann cells: inhibition by antibodies to NGF. NEURON. 1991; 7:265-75.

[29] Ahmed AM, Weller RO. The blood-nerve barrier and reconstitution of the perineurium following nerve grafting. Neuropathol Appl Neurobiol. 1979; 5:469-83.

[30] Parmantier E, Lynn B, Lawson D, et al. Schwann cell-derived Desert hedgehog controls the development of peripheral nerve sheaths. NEURON. 1999; 23:713-24.

[31] Webber CA, Christie KJ, Cheng C, et al. Schwann cells direct peripheral nerve regeneration through the Netrin-1 receptors, DCC and Unc5H2. GLIA. 2011; 59:1503-17.

[32] Soderblom C, Luo X, Blumenthal E, et al. Perivascular fibroblasts form the fibrotic scar after contusive spinal cord injury. J NEUROSCI. 2013; 33:13882-7.

[33] Sharghi-Namini S, Turmaine M, Meier C, et al. The structural and functional integrity of peripheral nerves depends on the glial-derived signal desert hedgehog. J NEUROSCI. 2006; 26:6364-76.

[34] Chang HY, Chi JT, Dudoit S, et al. Diversity, topographic differentiation, and positional memory in human fibroblasts. Proc Natl Acad Sci U S A. 2002; 99:12877-82.

[35] Que J, Cao Q, Sui T, et al. Effect of FK506 in reducing scar formation by inducing fibroblast apoptosis after sciatic nerve injury in rats. CELL DEATH DIS. 2013; 4:e526.

[36] Li B, Wang JH. Fibroblasts and myofibroblasts in wound healing: force generation and measurement. J TISSUE VIABILITY. 2011; 20:108-20.

[37] Chan JR, Cosgaya JM, Wu YJ, et al. Neurotrophins are key mediators of the myelination program in the peripheral nervous system. Proc Natl Acad Sci U S A. 2001; 98:14661-8.

[38] Yi S, Yuan Y, Chen Q, et al. Regulation of Schwann cell proliferation and migration by miR-1 targeting brain-derived neurotrophic factor after peripheral nerve injury. Sci Rep. 2016; 6:29121.

[39] Xiao J, Wong AW, Willingham MM, et al. BDNF exerts contrasting effects on peripheral myelination of NGF-dependent and BDNF-dependent DRG neurons. J NEUROSCI. 2009; 29:4016-22.

[40] Chernousov MA, Stahl RC, Carey DJ. Schwann cells use a novel collagen-dependent mechanism for fibronectin fibril assembly. J CELL SCI. 1998; 111 ( Pt 18):2763-77.

[41] Zhao X, Zhao G, Shi Z, et al. Low-intensity pulsed ultrasound (LIPUS) prevents periprosthetic inflammatory loosening through FBXL2-TRAF6 ubiquitination pathway. Sci Rep. 2017; 7:45779.

[42] Hsueh YY, Chang YJ, Huang TC, et al. Functional recoveries of sciatic nerve regeneration by combining chitosan-coated conduit and neurosphere cells induced from adipose-derived stem cells. BIOMATERIALS. 2014; 35:2234-44.

[43] Sorrell JM, Caplan AI. Fibroblasts-a diverse population at the center of it all. Int Rev Cell Mol Biol. 2009; 276:161-214. 